bound in loose leaf form, these additions can be clipped in as they become available. So far the Chiroptera, Primates, Rodentia, Carnivora and Artiodactyla are well represented, the other orders usually by three or four species, but the important Marsupialia by only one. Among the omissions, the cetacean is probably the most important order which has no representative.

The specimens all seem to be well preserved and with little distortion or damage. The standard of photography and reproduction is high. Ten linear dimensions (brain length, height and so on) are given for each specimen, and also brain and body weight, the sex (when known) and the method of fixation. Labelling is limited to quite obvious macroscopic structures and will be useful chiefly in relation to sulci and gyri in those brains where such features are evident. The short introductory text is given in both German and English, and a latinized terminology, internationally understood, is used for the parts of the brain. There is an extensive bibliography classified in sections for each of the mammalian orders.

This illustrated catalogue will be of value to taxonomists and, as Professor Stark points out in his Geleitwort, particularly to those interested in the evolution of brain morphology. In this context its value is increased by the fact that the brain is the only nonskeletal organ the form and subdivisions of which can be assessed, often with considerable accuracy, in fossil material from endocranial casts. It will provide also a necessary basis in macroscopic morphology for anyone beginning a study in an unfamiliar brain or indeed in comparative neuroanatomy in general. It is to be hoped that the additions needed to make it more complete will soon become available.

\section{F. GOLDBY}

\section{Insect Haemocytes}

The Cellular Defence Reactions of Insects. By George Salt. (Cambridge Monographs in Experimental Biology, No. 16.) Pp. vi +118 . (Cambridge University: London, October 1970.) 50s; \$7.50.

APART from the occasional devastating epidemic, insects usually seem to the human observer to enjoy excellent health. They thrive in a variety of highly contaminated environments, and they can withstand surgical operations of great severity without benefit of antibiotics. And although many viruses, bacteria, fungi and insect parasitoids can attack insects, these infections are often successfully thrown off. Their resistance is of two kinds. Immune responses, involving the formation of antibodies, are unknown in insects. Yet there are less specific serum responses which are of importance. These are to furnish the subject matter for a future monograph in this series. The present book describes, in elegant prose, the second line of defence: the defence interposed by the blood haemocytes to objects, animate or inanimate, that come to invade the haemocoel.

Smaller foreign particles entering the body cavity suffer phagocytosis. Larger masses such as a hymenopterous parasite of the "wrong" species or an implanted fragment of glass, are rapidly invested in a capsule of blood cells. This intriguing process, which has been studied experimentally by Salt over a number of years, involves much that is likely to interest the cell biologist as well as the entomologist : the adhesion of the cells to the "non-self" substratum; the secretion of extra-cellular matrix-perhaps a mucopolysaccharide - which facilitates cohesion; and finally, the striking flattening process that affects the cells arriving later on the capsule wall.

Why do the cells adhere in the first place? Salt concludes that "the wide range of particles and foreign bodies to which insect blood cells adhere can have no positive charactistic in common; they can only be alike in lacking something". Thus the tendency to adhere to surfaces is regarded as a general property, the failure to adhere the exception. Looking at the problem this way round avoids the complexities associated with "specificity" in vertebrates. Nevertheless, the exceptional surface properties which prevent the lodgment of haemocytes are still of great interest. Circulating haemocytes, the autologous connective tissue layer that lines the haemocoel and any parasite eggs or larvae adapted to the host species in question all possess this property. What this "something" is remains to be decided. But the problem is now neatly framed and awaits further investigation. The whole matter is placed in perspective in a final chapter in which insect and vertebrate defence reactions are compared and contrasted.

The voluminous literature on insect haemocytes, their types and functions, is not notable for its clarity. This lucid and stimulating survey is therefore most welcome.

A. D. LEES

\section{On the Ice Field}

The Chemical Physics of Ice. By N. H. Fletcher. (Cambridge Monographs on Physics.) Pp. $x+271$. (Cambridge University: London, February 1970.) $80 s$.

Professor Fletcher is to be warmly congratulated for writing this eminently readable monograph which I can recommend without hesitation. The book is intended principally for graduate students as an exemplification of the principles of chemical physics by a detailed study of a single material, ice.

The scope of the book is one that has not been attempted before in such detail. The first seventy pages describe the structure of the water molecule, ice $1 \mathrm{~h}$ and the high pressure polymorphs. The next fifty pages consider the structure of water and the mechanisms of the growth of crystals by nucleation and from the liquid and vapour phases. After a brief chapter on the thermal properties of the lattice, the closing 100 pages are concerned with the line and point defects in ice, and their role in mechanical and electrical behaviour.

This book confirms my belief that a carefully told exposition, starting right at the beginning, is more satisfying to any serious student than a book covering a wider field but held together with less coherence. In this way we can understand both the limitations and strength of the text. The limitations are that Fletcher's study of inter-related topics leaves the impression that there is little to doubt in "what is now generally regarded as understood and accepted about the chemical physics of ice". The impression is given that if there is a discrepancy between theory and observation, it is probably the experiments which must be improved. Thus, an all too simple picture may seem to emerge from the treatment. Fletcher has noted that the pace of ice physics has accelerated recently, and most of the references in the book are less than ten years old. The most recent work has tended to give a less clear picture, and Physics of Ice (Plenum: New York, 1969), the proceedings of the International Symposium at Munich in 1968 , could be read with profit by those who graduate from Fletcher's book.

The second purpose of the book is to provide a synoptic view of the properties of ice for those engaged in research in glaciology, cloud physics and other related fields. Certainly reference volumes for glaciologists dismiss summarily the aspects of ice discussed by Fletcher, and so the book should interest them. Fletcher does not attempt to discuss the flow of glaciers (the creep of polycrystalline ice being concluded in half a page), yet correctly spends fourteen pages introducing and discussing mechanical relaxation in ice, as this is intimately related to the unique crystal structure of ice.

SI units would have been preferred in the text, and the paragraph on regelation on page 196 seems misplaced. The book is extremely well written and will certainly serve for long as an introduction to any branch of ice physics in which the reader has no specialized knowledge. J. G. PAREN 